\title{
Magnetic fields above the surface of a superconductor with internal magnetism
}

\author{
Hendrik Bluhm* \\ Department of Physics, Stanford University, Stanford, CA 94305
}

\begin{abstract}
The author presents a method for calculating the magnetic fields near a planar surface of a superconductor with a given intrinsic magnetization in the London limit. He computes solutions for various magnetic domain boundary configurations and derives relations between the spectral densities of the magnetization and the resulting field in the vacuum half space, which are useful if the magnetization can be considered as a statistical quantity and its features are too small to be resolved individually. The results are useful for analyzing and designing magnetic scanning experiments. Application to existing data from such experiments on $\mathrm{Sr}_{2} \mathrm{RuO}_{4}$ show that a domain wall would have been detectable, but the magnetic field of randomly oriented small domains and small defects may have been smaller than the experimental noise level.
\end{abstract}

PACS numbers: 74.25.Ha, 74.20.De, 75.70.-i, 74.70.Pq

\section{INTRODUCTION}

Starting with the discovery of ferromagnetic order in the superconductors $\mathrm{HoMo}_{6} \mathrm{~S}_{8} \frac{1,2}{2}$ and $\mathrm{ErRh}_{4} \mathrm{~B}_{4}{ }^{\frac{3}{4}}$ about three decades ago, there has been increasing interest in superconductors with an intrinsic magnetization. In the above cases and some of the $\mathrm{RNi}_{2} \mathrm{~B}_{2} \mathrm{C}$ compounds $(\mathrm{R}=$ rare earth), the magnetization is due to localized moments of the rare earth ions and coexists with superconductivity in some temperature range. In other materials, the conduction electrons may not only superconduct, but also carry some magnetization. A prominent example is $\mathrm{Sr}_{2} \mathrm{RuO}_{4}{ }^{4}$, which is believed to have a complex, time reversal symmetry breaking $p$-wave order parameter,,$\frac{5}{,}$ so that the orbital angular momentum of the Cooper pairs creates a magnetic moment. This is theoretically expected to cause edge currents at sample boundaries and domain walls 6.7 A similar effect has been suggested to occur in the $d$-wave superconductor $\mathrm{Na}_{x} \mathrm{CoO}_{2} \cdot y \mathrm{H}_{2} \mathrm{O} .8$

The traditional experimental techniques for studying magnetic ordering phenomena are bulk probes such as muon spin rotation $(\mu \mathrm{SR})$ or (spin polarized) small angle neutron scattering (SANS). An alternative approach is to use magnetic scanning techniques such scanning Hall probe microscopy (SHPM) $\stackrel{9}{\underline{9}}$ scanning SQUID microscopy $\stackrel{10}{2}$ magnetic force microscopy $(\mathrm{MFM})^{11.12}$ and magneto-optical techniques 13 These techniques measure the magnetic field some small distance above the surface of the sample as a function of position. In many cases, their resolution, which is limited by the probe size and probe-sample distance, does not quite reach the length scales typical for the magnetic structure. For example, one finds evidence for oscillatory magnetic order with a sub-penetration depth length scale in $\mathrm{HoMo}_{6} \mathrm{~S}_{8}{ }^{1,2}$ and $\mathrm{ErRh}_{4} \mathrm{~B}_{4}{ }^{3}$. For larger wave lengths, the Meissner effect in the coexisting state would screen the field and thus suppress magnetic interactions and destabilize the magnetic order. A similar situation has been observed in the superconductor $\mathrm{ErNi}_{2} \mathrm{~B}_{2} \mathrm{C}$ using SHPM 14 The direct observation of the magnetic fields generated by edge currents or domain walls in $\mathrm{Sr}_{2} \mathrm{RuO}_{4}$ is an ongoing effort which has not produced any evidence so $\operatorname{far}^{15,16}$.

To plan such scanning experiments and to interpret the resulting data, it is important to understand to what extent the magnetic field generated by a spatially varying magnetization inside the sample is propagated to the probe. In normal materials, this is a straightforward magnetostatics problem. In superconducting samples, however, it is complicated by the Meissner screening. In this work, I present solutions for an infinite planar sample surface by incorporating the presence of a magnetization $\mathbf{M}(\mathbf{x})$ into a London model and solving the resulting equations, using a 2D Fourier transform. This approach follows earlier theoretical work on superconductors with an internal magnetization due to localized magnetic moments, $, 17,18,19,20$ but should also apply if the magnetism is of different origin, such as a spin or orbital moment of the Cooper pairs. To consider the practically very likely case where the limited measurement resolution leads to significant averaging over several domains or other features with some degree of randomness, I present a spectral analysis. The resulting relations were employed for the analysis of the data in Ref. 14 and may also be used to analyze null results where no field variation is detectable at the experimental noise level. A similar analysis may also be useful for interpreting surface sensitive $\mu \mathrm{SR}$ experiments, which only average over a thin layer at the sample surface 21 .

The paper is organized as follows: In section II] I derive the main equations of our model from a generalized Ginzburg-Landau (GL) functional. Those equations are solved in a general framework in section III. In section IV. I discuss simple domain wall and dipole configurations as examples. Relations between the spectral densities of the magnetization and the magnetic field in vacuum are computed in section V Section VI applies the results to recent magnetic scanning work ${ }^{15}$ on $\mathrm{Sr}_{2} \mathrm{RuO}_{4}$. 


\section{MODEL}

In order to fully describe the interplay between magnetism and superconductivity in a phenomenological approach, the magnetization $\mathbf{M}$ and the superconducting order parameter $\psi$ have to be computed self consistently, taking mutual interactions into account. This has been done in Refs. 17, 18, 19,20 using the generalized GL functional

$$
\begin{aligned}
F[\psi, \mathbf{M}, \mathbf{A}]= & \int d^{3} \mathbf{r}\left[\frac{1}{2} a|\psi|^{2}+\frac{1}{4} b|\psi|^{4}+\frac{\hbar^{2}}{2 m^{*}}\left|\left(i \nabla+\frac{2 e}{\hbar c} \mathbf{A}\right) \psi\right|^{2}\right. \\
& \left.+\frac{1}{2} \alpha|\mathbf{M}|^{2}+\frac{1}{4} \beta|\mathbf{M}|^{4}+\frac{1}{2} \gamma^{2}|\nabla \mathbf{M}|^{2}+\frac{1}{8 \pi} \mathbf{B}^{2}-\mathbf{B} \cdot \mathbf{M}\right],
\end{aligned}
$$

where $\mathbf{B}=\nabla \times \mathbf{A}$. In this work, I will assume this task to be partially solved by starting with a given $\mathbf{M}(\mathbf{x})$ and computing the resulting magnetic field, taking shielding currents into account. This approach is clearly justified if the magnetic energy scale is much larger than the superconducting one, so that the effect of superconductivity on magnetism can be neglected. However, it is also reasonable if the result of a self consistent calculation for $\mathbf{M}$ is (approximately) known, for example from bulk calculations, and one is mainly concerned with the effect of the reduced screening at the surface on the observable field. The errors introduced by this treatment will then primarily be due to the effect of the surface and the modified screening on $\mathbf{M}$.

Writing the order parameter as $\psi=|\psi| e^{i \phi}$ and introducing the London penetration depth defined by $\lambda^{-2}=$ $4 \pi\left(|\psi|^{2} / m^{*}\right) e^{* 2} / c^{2}$, variation of Eq. (1) with respect to A leads to

$$
\nabla \times(\nabla \times \mathbf{A}-4 \pi \mathbf{M})+1 / \lambda^{2} \mathbf{A}=\left(\Phi_{0} / 2 \pi \lambda^{2}\right) \nabla \phi .
$$

By performing a line integral over a closed loop after multiplying by $\lambda^{2}$ and using the Stokes theorem, one obtains

$$
\nabla \times\left(\lambda^{2} \nabla \times \mathbf{B}\right)+\mathbf{B}=4 \pi \nabla \times\left(\lambda^{2} \nabla \times \mathbf{M}\right)+\Phi_{0} \mathbf{f} .
$$

$\mathbf{f}$ is a sum of $2 \mathrm{D} \delta$ - functions representing vortex cores, which will be ignored in the following. This result can be obtained directly by treating the supercurrent density $\mathbf{j}_{s}$ as macroscopic current in the macroscopic Maxwell equation and thus substituting $\nabla \times(\mathbf{B}-4 \pi \mathbf{M})=4 \pi / c \mathbf{j}_{s}$ into the London equation $\nabla \times\left(4 \pi \lambda^{2} / c \mathbf{j}_{s}\right)+\mathbf{B}=\Phi_{0} \mathbf{f}$. Although the above is valid for a spatially varying superfluid density $n_{s}=|\psi|^{2} / m^{*}$, I will assume $\lambda$ to be constant, which leads to the more familiar form

$$
\nabla \times \nabla \times \mathbf{B}+1 / \lambda^{2} \mathbf{B}=4 \pi \nabla \times \nabla \times \mathbf{M}
$$

I assume the magnetic superconductor to occupy the lower half space $z<0$. In vacuum $(z>0)$, the magnetic field must satisfy $\nabla \cdot \mathbf{B}=0$ and $\nabla \times \mathbf{B}=0$. At the interface, the normal component of $\mathbf{B}$ and the tangential component of $\mathbf{H}=\mathbf{B}-4 \pi \mathbf{M}$ must be continuous.
Note that $\mathbf{M}$ enters Eq. 3 only through the microscopic current density $\mathbf{j}_{\mathbf{M}}=c \nabla \times \mathbf{M}$. Thus, one may also start directly from an intrinsic current density $\mathbf{j}_{\mathbf{M}}$ rather than $\mathbf{M}$, which is more natural if an edge current is known from microscopic calculations, for example. The appearance of $\mathbf{M}$ in the tangential boundary condition can also be eliminated by replacing it with a discontinuity in $\mathbf{M}$ just below the surface.

\section{SOLUTION}

In Ref. 22, the field geometry of a vortex penetrating the surface of an anisotropic superconductor for a general orientation of the vortex and the main axis of the effective mass tensor with respect to the interface has been computed. In the vortex problem, the right hand side appearing in the London equation Eq. (3) is a $2 \mathrm{D}$ delta function instead of the magnetization term. I use the same technique, but only present the calculations for the isotropic case for the sake of simplicity.

The Maxwell equations in vacuum can be satisfied by writing the magnetic field as $\mathbf{B}=-\nabla \Phi$ with $-\nabla^{2} \Phi=0$. A suitable solution has to be matched to a solution of Eq. (3) at $z=0$. I solve this problem using a 2D Fourier transform $(\mathrm{FT})$ in the $x y$-plane, i.e. by writing a function $A(x, y, z)$ as $A\left(\mathbf{r}_{\|,}, z\right)=(2 \pi)^{-2} \int d^{2} \mathbf{k} \tilde{A}(\mathbf{k}, z) e^{i \mathbf{k} \cdot \mathbf{r}_{\|} \text {, }}$ with $\mathbf{r}_{\|}=(x, y)$ and $\mathbf{k}=\left(k_{x}, k_{y}\right)$. (Note that I use the same symbol for 2 and 3 dimensional vectors, implying that the $z$-component vanishes for the latter.) For $z<0$, the field $\mathbf{B}$ is decomposed as $\mathbf{B}_{0}+\mathbf{B}_{1} \cdot \mathbf{B}_{0}$ is a particular solution of the inhomogeneous London equation (3) in full space with boundary conditions at infinity and with the right hand side suitably extended to $z>0 . \mathbf{B}_{1}$ is a general homogeneous solution chosen to satisfy the matching condition at the interface. Under the 2D FT, Eq. (3) transforms into $\left(k^{2}+1 / \lambda^{2}-\partial^{2} / \partial z^{2}\right) \tilde{\mathbf{B}}_{1}=0$, so that $\tilde{\mathbf{B}}_{1}(\mathbf{k}, z)=\mathbf{B}_{\mathbf{k}} e^{K z}$ with $K=\sqrt{k^{2}+1 / \lambda^{2}}$. In vacuum, $-\nabla^{2} \Phi=0$ has the solutions $\tilde{\Phi}(\mathbf{k}, z)=\Phi_{\mathbf{k}} e^{-k z}$ so that at $z>0, \tilde{\mathbf{B}}(\mathbf{k}, z)=\left(-i \mathbf{k}+k \hat{\mathbf{e}}_{z}\right) \Phi_{\mathbf{k}} e^{-k z}$. 
Hence, $\nabla \cdot \mathbf{B}_{1}=0$ together with the $\hat{\mathbf{e}}_{z}, \hat{\mathbf{k}}$ and $\hat{\mathbf{k}} \times \hat{\mathbf{e}}_{z}$ components of the continuity conditions for $\mathbf{B}$ and $\mathbf{H}=$ $\mathbf{B}-4 \pi \mathbf{M}$ at $z=0 \mathrm{read}$

$$
\begin{aligned}
0 & =i \mathbf{k} \cdot \mathbf{B}_{\mathbf{k}}+K \hat{\mathbf{e}}_{z} \cdot \mathbf{B}_{\mathbf{k}} \\
k \Phi_{\mathbf{k}} & =\hat{\mathbf{e}}_{z} \cdot\left(\mathbf{B}_{\mathbf{k}}+\tilde{\mathbf{B}}_{0}(\mathbf{k}, 0)\right) \\
-i k \Phi_{\mathbf{k}} & =\hat{\mathbf{k}} \cdot\left(\mathbf{B}_{\mathbf{k}}+\tilde{\mathbf{B}}_{0}(\mathbf{k}, 0)-4 \pi \tilde{\mathbf{M}}(\mathbf{k}, 0)\right) \\
0 & =\left(\hat{\mathbf{k}} \times \hat{\mathbf{e}}_{z}\right) \cdot\left(\mathbf{B}_{\mathbf{k}}+\tilde{\mathbf{B}}_{0}(\mathbf{k}, 0)-4 \pi \tilde{\mathbf{M}}(\mathbf{k}, 0)\right) .
\end{aligned}
$$

The last equation for the in-plane transverse component of $\mathbf{B}_{\mathbf{k}}$ is already decoupled and can be dropped if only the vacuum field is to be computed. Solving the first three equations for $\Phi_{\mathbf{k}}$ leads to

$$
k(K+k) \Phi_{\mathbf{k}}=K \hat{\mathbf{e}}_{z} \cdot \tilde{\mathbf{B}}_{0}(\mathbf{k}, 0)+i \mathbf{k} \cdot\left(\tilde{\mathbf{B}}_{0}(\mathbf{k}, 0)-4 \pi \tilde{\mathbf{M}}(\mathbf{k}, 0)\right)
$$

If the 2D FT of the inhomogeneous solution, $\tilde{\mathbf{B}}_{0}$, cannot be obtained directly, the $3 \mathrm{D}$ FT $\mathbf{B}_{0}(\mathbf{q})$ of $\mathbf{B}_{0}(\mathbf{r})$ can be obtained from $\mathbf{M}(\mathbf{q})$ by solving the $3 \times 3$ linear system

$$
-\mathbf{q} \times\left(\mathbf{q} \times \mathbf{B}_{0}(\mathbf{q})\right)+1 / \lambda^{2} \mathbf{B}_{0}(\mathbf{q})=-4 \pi \mathbf{q} \times(\mathbf{q} \times \mathbf{M}(\mathbf{q}))
$$

The solenoidal condition $\nabla \cdot \mathbf{B}_{0}=0$ will always hold as $\mathbf{q} \cdot \mathbf{B}(\mathbf{q})=\lambda^{2} \mathbf{q} \cdot[\mathbf{q} \times(\mathbf{q} \times \mathbf{B}(\mathbf{q}))-4 \pi \mathbf{q} \times(\mathbf{q} \times \mathbf{M}(\mathbf{q}))]=$ 0 . The $2 \mathrm{D}$ FT at $z=0$ is then simply $\tilde{\mathbf{B}}_{0}(\mathbf{k}, 0)=$ $(1 / 2 \pi) \int d q_{z} \mathbf{B}_{0}(\mathbf{q})$ with $\mathbf{q}=\mathbf{k}+q_{z} \hat{\mathbf{e}}_{z}$. While this approach to the inhomogeneous problem is very convenient for numerical evaluation and can be generalized to the anisotropic case (cf. Ref. 22), it is useful to derive an explicit solution. The component of $\mathbf{M}$ parallel to $\mathbf{q}$ does not contribute to $\mathbf{q} \times \mathbf{M}$. For components of $\mathbf{B}_{0}$ and $\mathbf{M}$ orthogonal to $\mathbf{q}, \mathbf{q} \cdot \mathbf{B}_{0}=0$ automatically and the vector products simplify to scalar multiplication. By decomposing $\mathbf{M}$ and $\mathbf{B}_{0}$ into components along the unit vectors $\hat{\mathbf{e}}_{x y}=\mathbf{q} \times \hat{\mathbf{e}}_{z} /\left|\mathbf{q} \times \hat{\mathbf{e}}_{z}\right|$ orthogonal to $\mathbf{q}$ in the $x y$-plane and $\hat{\mathbf{e}}_{\perp}=\hat{\mathbf{q}} \times \hat{\mathbf{e}}_{x y}$, the inhomogeneous solution thus simplifies to

$$
\begin{aligned}
B_{0, \perp} & =\frac{k^{2}+q_{z}^{2}}{1 / \lambda^{2}+k^{2}+q_{z}^{2}} 4 \pi M_{\perp} \\
B_{0, x y} & =\frac{k^{2}+q_{z}^{2}}{1 / \lambda^{2}+k^{2}+q_{z}^{2}} 4 \pi M_{x y}
\end{aligned}
$$

To evaluate Eq. (41), the inverse z-FT must be carried out in order to obtain the values at $z=0$, unless $\mathbf{M}$ is $z$ independent. It turns out that $B_{0, x y}$ does not enter Eq. (44) because of the dot product with k. Projecting $B_{0, \perp}$ onto the $z$ and $\hat{\mathbf{k}}$ direction, substituting Eq. (5), and expressing everything in terms of $M_{z}$ and $\hat{\mathbf{k}} \cdot \mathbf{M}$ using $M_{\perp}=\frac{-k}{\sqrt{q_{z}^{2}+k^{2}}} M_{z}+\frac{q_{z}}{\sqrt{q_{z}^{2}+k^{2}}} \hat{\mathbf{k}} \cdot \mathbf{M}$ leads to

$$
\begin{aligned}
k(K+k) \Phi_{\mathbf{k}} & =2 \int d q_{z} \frac{k^{2}\left(K-i q_{z}\right)}{1 / \lambda^{2}+k^{2}+q_{z}^{2}} M_{z}(\mathbf{q}) \\
& -2 i \int d q_{z} \frac{K\left(K-i q_{z}\right)}{1 / \lambda^{2}+k^{2}+q_{z}^{2}} \mathbf{k} \cdot \mathbf{M}(\mathbf{q})
\end{aligned}
$$

The $i q_{z}$ terms in the numerator of the fractions can be dropped if one uses the convention that $\mathbf{M}$ is extended to $z>0$ as an even function so that those terms do not contribute to the integrals. Eq. (6) can be summarized qualitatively as follows: For in-plane components of $\mathbf{M}$, the source of $\mathbf{B}$ outside the superconductor is the divergence of $\mathbf{M}$ averaged over one penetration depth below the surface. For the normal component, an additional derivative is taken, thus increasing the multipole order of the vacuum field by one. The small $k$ components of the field are just those resulting from the subsurface magnetization and its image obtained by reflection about a plane $\lambda$ below the surface.

I would like to point out that for the solution method to work as described, the interface must be planar and $\lambda^{2}$ should be constant. $\mathbf{M}$ on the contrary can be an arbitrary function. However, if $\nabla \times \mathbf{M}=0$ so that $\mathbf{B}_{0}=0$ solves Eq. (2), the requirement that $\lambda^{2}$ may not depend on $z$ can be dropped at the expense of solving a more complicated ordinary differential equation instead of the Laplace equation to obtain $\tilde{\mathbf{B}}_{1}(\mathbf{k}, z)$. For example, discontinuities in $\lambda^{2}$ as a function of $z$ could be treated by matching additional continuity conditions. A dependence of $\lambda^{2}$ on $x$ or $y$ on the other hand would mix different $\mathbf{k}$ components and thus generally forbid a simple analytic solution.

\section{EXAMPLES}

\section{A. Discussion of Table I}

As examples for various simple, representative configurations in $\mathbf{M}$, I have calculated the field of ferromagnetic domain walls, where $\mathbf{M}$ changes sign, and dipoles at the surface. For $z \gg \lambda$, simple approximate expressions in real space can be obtained. The results are shown in table प. The approximations are based on the fact that for $z \gg \lambda$, only $k_{x} \ll 1 / \lambda$ contributes to the Fourier integral $B_{z}(x, z)$. To second order, $1 /(K+|k|) \approx \lambda e^{-|k| \lambda}$ and $K \approx(1 / \lambda)\left(1+(k \lambda)^{2} / 2\right)$. Thus, the approximations in table \are good to three and two orders beyond leading order in $k$ for cases (1),(3) and (2), (4), respectively. In the following, I discuss the far fields obtained from these approximations. To understand those, it is useful to recall that for magnetostatic problems in the absence of macroscopic or supercurrents, $-\nabla^{2} \Phi=-4 \pi \nabla \cdot \mathbf{M}$. Thus, $\nabla \cdot \mathbf{M}$ acts as a magnetic charge by analogy with electrostatics. The field of a discontinuity of the in-plane component of $\mathbf{M}$ [case (1)] is just twice that of a magnetically charged line with linear charge density $4 \pi 2 M \lambda$ situated $\lambda$ below the surface. It can be understood as the charge density due to the discontinuity of $2 M$ in the magnetization, which is screened by supercurrents over one penetration depth. The additional factor two formally comes from the extension of $M$ to $z>0$. For a discontinuity in the normal component $M_{z}$ [case (2)], one obtains the dipole field of two lines of opposite magnetic charge 
with a charge density $M \lambda$ as above and a separation of $\lambda$. Again, this can be understood as the screened field of the discontinuity in the magnetization occurring at the surface.

The localized dipole pointing into or out of the surface [case (3)] has a a quadrupole field. An in-plane moment [case (4)] on the contrary has a dipole field to leading order. For a dipole chain, i.e. $\mathbf{M}=\tilde{m} \delta(x) \delta(y) \hat{\mathbf{e}}_{z}$, I obtain the same results as for a single dipole oriented in the $z$ direction $[\operatorname{case}(3)]$ with $m=\lambda \tilde{m}$. The same analogy can be drawn for case (4).

It is also of interest to consider a configuration where a magnetization $M \hat{\mathbf{e}}_{z}$ is localized over a width $w$ around $x=0$. This situation may be encountered at an antiferromagnetic domain wall, where canting of antiferromagnetically ordered in-plane moments produces a local net outof-plane magnetization. The corresponding exact solution is a superposition of two solutions for normal discontinuities with opposite signs, shifted by $w$. If $w$ is smaller than all other length scales, $M(x)$ can be replaced by a delta function: $M(x) \approx\left(\int M\left(x^{\prime}\right) d x^{\prime}\right) \delta(x)=w M \delta(x)$. The resulting field is then simply the derivative of that of a discontinuity in $M_{z}$, i.e. for $z \gg \lambda$,

$$
B_{z}(x, z) \approx-4 M \lambda^{2} w \frac{d^{2}}{d x^{2}} \frac{(z+\lambda)}{(z+\lambda)^{2}+x^{2}}
$$

\section{B. Periodic configurations}

If the magnetization is periodic, the Fourier integrals turn into Fourier sums. If the period $L$ is large $(L \gtrsim 2 \pi z)$, features with size comparable to $L$ can be resolved in each unit cell individually and look similar to a solution obtained from a constant continuation of $\mathbf{M}$ outside that cell. For shorter periods however, the superposition of many such single cell solutions largely cancels out. Formally, this follows from the fact that the wave vector $k$ takes only integer multiples of $2 \pi / L$. Therefore, the dominant contributions at $k \lesssim 1 / z$ considered in table I are not present an the leading term becomes that of the lowest wave vector $k=2 \pi / L$. This results in an exponential suppression by $e^{-2 \pi z / L}$ of the lowest harmonic of the field variation at a height $z$ above the surface and all higher harmonics being negligible for $z \gtrsim L$.

\section{Effect of smoothing}

As the exponential cutoff in the inverse FT becomes increasingly sharper for larger $z$, the far field from any feature of finite size in the magnetization will always be determined by the lowest non-vanishing power of $k$ in $k \Phi_{\mathbf{k}}$ for sufficiently large $z$. For a smooth domain wall, $\mathbf{M}(\mathbf{q})$ has less weight at large $\mathbf{q}$ compared to a sharp discontinuity of equal magnitude, but the values at small $\mathbf{q}$ are affected little. Thus, the asymptotic results for sharp discontinuities in $\mathbf{M}$ are still valid if $\mathbf{M}$ changes smoothly over a width $w$ as long as $z \gg w$. For example, the far field of a single domain boundary does not depend on the length scale over which the magnetization changes in the $x y$-plane, but only on the difference between the asymptotic values of $M$ on both sides. Hence, the approximate results in table $\Pi$ are of rather general validity. For a graphic illustration, see Fig. 2, which is discussed in Sec. VI. The situation is slightly different for a nontrivial $z$ dependence of $\mathbf{M}$ : the $q_{z}$-cutoff is always determined by the $1 /\left(1+\left(\lambda q_{z}\right)^{2}\right)$ factor for small $k$. In real space, this corresponds to an exponentially weighted average over a distance of $\lambda$ below the surface.

\section{SPECTRAL ANALYSIS}

The resolution of currently available magnetic imaging techniques is often not sufficient to resolve an actual domain structure. In practice, averaging occurs both due to the imaging height $z$ and the finite sensor size. Here, I will only consider the more universal height effect. For a height larger than the typical domain size, the magnetic field represents an average over several domains. As shown in the previous section, this would lead to an exponential suppression for periodic configurations. However, domains will usually not be strictly periodic, but have some distribution of size. Therefore, a statistical description is most adequate. Assuming a given correlation function and thus spectrum of the magnetization $\mathbf{M}(\mathbf{r})$, I compute the spectrum of the resulting magnetic field.

The spectral function of two functions $f(\mathbf{r})$ and $g(\mathbf{r})$ in $d$ dimensions, $S_{f g}(\mathbf{q})=\int d^{d} \mathbf{r}^{\prime}\left\langle f(\mathbf{r}) g\left(\mathbf{r}+\mathbf{r}^{\prime}\right)\right\rangle e^{-i \mathbf{r}^{\prime} \cdot \mathbf{q}}$, satisfies the relation $\left\langle f(\mathbf{q})^{*} g\left(\mathbf{q}^{\prime}\right)\right\rangle=(2 \pi)^{d} \delta^{d}\left(\mathbf{q}-\mathbf{q}^{\prime}\right) S_{f g}(\mathbf{q})$ Here, $\langle\cdot\rangle$ stands for an ensemble average over different realizations of $f$ and $g$. It is implicit to this definition that the correlator $\left\langle f(\mathbf{r}) g\left(\mathbf{r}+\mathbf{r}^{\prime}\right)\right\rangle$ is independent of $\mathbf{r}$.

It is convenient to introduce the propagation coefficients

$$
\begin{aligned}
c_{\mathbf{q}, z} & =2 k^{2} \lambda^{2} \gamma_{\mathbf{q}} \\
c_{\mathbf{q}, \alpha} & =-2 i k_{\alpha} K \lambda^{2} \gamma_{\mathbf{q}} \quad(\alpha=x, y) \\
\gamma_{\mathbf{q}} & =\frac{\left(K-i q_{z}\right) e^{-k z}}{\lambda^{2}(k+K)\left(1 / \lambda^{2}+k^{2}+q_{z}^{2}\right)}
\end{aligned}
$$

and rewrite Eq. (6), using $\tilde{B}_{z}(\mathbf{k}, z)=k \Phi_{\mathbf{k}} e^{-k z}$, as $B_{z}\left(z_{0}, \mathbf{k}\right)=\int d q_{z} \sum_{\alpha} c_{\mathbf{q}, \alpha} M_{\alpha}(\mathbf{q})$. It follows that

$$
\begin{aligned}
S_{B_{z}}(\mathbf{k}, z) & =2 \pi \int d q_{z} \sum_{\alpha, \beta} c_{\mathbf{q}, \alpha}^{*} c_{\mathbf{q}, \beta} S_{M, \alpha \beta}(\mathbf{q}) \\
& =2 \pi \int d q_{z} \sum_{\alpha}\left|c_{\mathbf{q}, \alpha}\right|^{2} S_{M, \alpha \alpha}(\mathbf{q}) \\
& +4 \pi \int d q_{z} \sum_{\alpha \neq \beta} \operatorname{Re}\left(c_{\mathbf{q}, \alpha}^{*} c_{\mathbf{q}, \beta} S_{M, \alpha \beta}(\mathbf{q})\right)
\end{aligned}
$$




\begin{tabular}{|c|c|c|c|}
\hline Case & $\mathbf{M}(\mathbf{r})$ & $B_{z}$ (exact) & $B_{z}$ (approximate) \\
\hline \hline$(0)$ & $M(x) \hat{\mathbf{e}}_{y}$ & 0 & $-4 M \int d k \lambda e^{i k x} e^{-|k|(z+\lambda)}=-8 M \lambda \frac{z+\lambda}{(z+\lambda)^{2}+x^{2}}$ \\
\hline$(1)$ & $M \operatorname{sgn}(x) \hat{\mathbf{e}}_{x}$ & $-4 M \int d k \frac{1}{|k|+K} e^{i k x} e^{-|k| z}$ & $4 M \int d k(-i k) \lambda^{2} e^{i k x} e^{-|k|(z+\lambda)}=-8 M \lambda^{2} \frac{d}{d x} \frac{z+\lambda+\lambda)^{2}+x^{2}}{(z+K)}$ \\
\hline$(2)$ & $M \operatorname{sgn}(x) \hat{\mathbf{e}}_{z}$ & $4 M \int d k \frac{-i k K e^{i k x} e^{-|k| z}}{\left(1 / \lambda^{2}+k^{2}\right)(|k|+K)}$ & $2 m \int_{0}^{\infty} d k k^{3} \lambda J_{0}(k x) e^{-k(z+\lambda)}=2 m \lambda \frac{6(z+\lambda)^{3}-9 r^{2}(z+\lambda)}{\left((z+\lambda)^{2}+r^{2}\right)^{7 / 2}}$ \\
\hline$(3)$ & $m \hat{\mathbf{e}}_{z} \delta^{3}(\mathbf{r})$ & $2 m \int_{0}^{\infty} d k \frac{k^{3}}{k+K} J_{0}(k r) e^{-k z}$ & $2 m \cos \varphi \int_{0}^{\infty} d k k^{2} J_{1}(k r) e^{-k(z+\lambda)}=2 m \cos \varphi \frac{3 r(z+\lambda)}{\left(r^{2}+\left(z+\lambda^{2}\right)\right)^{5 / 2}}$ \\
\hline
\end{tabular}

TABLE I: Exact and approximate expressions for the magnetic field above the superconductor for domain walls and dipoles with an in-plane and out-of-plane magnetization. In case (4), $\varphi$ is the angle between the $x$-axis and $\mathbf{r}$. The approximate expressions in the last column are valid for $z \gg \lambda$.

I have used $S_{M, \alpha \beta}$ as a short hand notation for the spectral function $S_{M \alpha M \beta}$ of two different components of $\mathbf{M}$. Similar expressions can be written down for spectral densities involving other components of $\mathbf{B}$.

Because of the presence of the surface, the assumption of translational invariance in the $z$-direction implicit to the definition of $S_{M}$ is by no means trivial. If the presence of the surface does not affect the structure of $\mathbf{M}$ too much, and the range of the surface influence is much shorter than $\lambda$, most of it should average out because $B_{z}$ is sensitive to what happens within a layer of thickness $\lambda$ below the surface. However, the $z$-invariance is only required in order to define spectral functions $S_{M, \alpha \beta}$. If the interface (or other effects) do break the $z$-invariance of $\left\langle M_{\alpha}(\mathbf{r}) M_{\beta}\left(\mathbf{r}+\mathbf{r}^{\prime}\right)\right\rangle$ so that it depends on both $z$ and $z^{\prime}$, it is still possible to derive an expression for $S_{B_{z}}$ similar to Eq. (10), however involving a double integral over $q_{z}$.

A statistical analysis will be most relevant when the measurement height $z$ is much larger than any of the intrinsic length scales of the variation of $\mathbf{M}$, i.e. too large to resolve individual features. In this case, $S_{M}\left(\mathbf{k}+q_{z} \hat{\mathbf{e}}_{z}\right)$ will not have a strong $\mathbf{k}$ dependence in the small $\mathbf{k}$ region surviving the $e^{-k z}$ cutoff and can be approximated by $S_{M}\left(q_{z} \hat{\mathbf{e}}_{z}\right)$. For $z \gg \lambda$, a similar approximation can be made for the propagation coefficients $c_{\mathbf{q}, \alpha}$ and $\gamma_{\mathbf{q}}$ :

$$
\begin{aligned}
c_{\mathbf{q}, \alpha} & \approx-2 i k_{\alpha} \lambda \gamma_{\mathbf{q}} \quad(\alpha=x, y) \\
\left|\gamma_{\mathbf{q}}\right|^{2} & \approx \frac{e^{-2 k(z+\lambda)}}{1+q_{z}^{2} \lambda^{2}}
\end{aligned}
$$

In any case, the properties of $\mathbf{M}$ only enter via the integrals

$$
\int d q_{z} \frac{1}{1+k^{2} \lambda^{2}+q_{z}^{2} \lambda^{2}} S_{M, \alpha \beta}(\mathbf{q})
$$

As argued above, it will often be a good approximation to set $\mathbf{k}=0$. In many cases, $S_{M}\left(q_{z} \hat{\mathbf{e}}_{z}\right)$ will have a peak at some wave vector $q_{0}$ (and consequently at $-q_{0}$ ), similar to the illustration in Fig. 1. For simplicity, I assume that there is only one such maximum. A finite $q_{0}$ is a signature of an oscillatory behavior of $\mathbf{M}$. The width of the peak corresponds to the inverse coherence length of the oscillation or the correlation length for $q_{0}=0$.

The integral (13) can be approximated further in two limiting cases. If the coherence length of $\mathbf{M}$ along the $z$ direction is much larger than $\lambda$, then $S_{M}\left(q_{z} \hat{\mathbf{e}}_{z}\right)$ is sharply peaked, and the kernel $1 /\left(1+q_{z}^{2} \lambda^{2}\right)$ can be replaced by $1 /\left(1+q_{0}^{2} \lambda^{2}\right)$ and pulled out of the integral. In the opposite limit, the peak in $S_{M}\left(q_{z} \hat{\mathbf{e}}_{z}\right)$ is much wider than $1 / \lambda$ so that the $q_{z}$ dependence of $S_{M}$ can be neglected entirely and one obtains

$$
\int d q_{z} \frac{1}{1+q_{z}^{2} \lambda^{2}} S_{M, \alpha \beta}(\mathbf{q}) \approx(\pi / \lambda) S_{M}(0)
$$

Assuming that the $\alpha$-component of $\mathbf{M}$ dominates, the respective diagonal term of Eq. (10) takes the form

$$
S_{B_{z}}(\mathbf{k})=\left(8 \pi^{2} / \lambda\right)\left(k_{\alpha} \lambda\right)^{2} e^{-2 k(z+\lambda)} S_{M, \alpha \alpha}(0)
$$

for $\alpha=x, y$. If the $M_{z}$ component is dominant, $\left(k_{\alpha} \lambda\right)^{2}$ in the prefactor must be replaced by $(k \lambda)^{4}$. Similar expressions can be written down for the off-diagonal components of $S_{M, \alpha \beta}$. For an illustration of the relation between a measured $B_{z}$ and its spectral function, the reader is referred to Ref. 14. Integration leads to simple expressions for $\left\langle B_{z}^{2}\right\rangle=(2 \pi)^{-2} \int d^{2} \mathbf{k} S_{B_{z}}(\mathbf{k})$ :

$$
\begin{aligned}
& \left\langle B_{z}^{2}\right\rangle=\frac{3 \pi}{4} \frac{\lambda}{(z+\lambda)^{4}} S_{M, \alpha \alpha}(0) \quad \text { for } \alpha=x, y \\
& \left\langle B_{z}^{2}\right\rangle=\frac{15 \pi}{2} \frac{\lambda^{3}}{(z+\lambda)^{6}} S_{M, z z}(0) .
\end{aligned}
$$

Those expressions can be used to estimate the signal expected in a scanning experiment or to estimate 

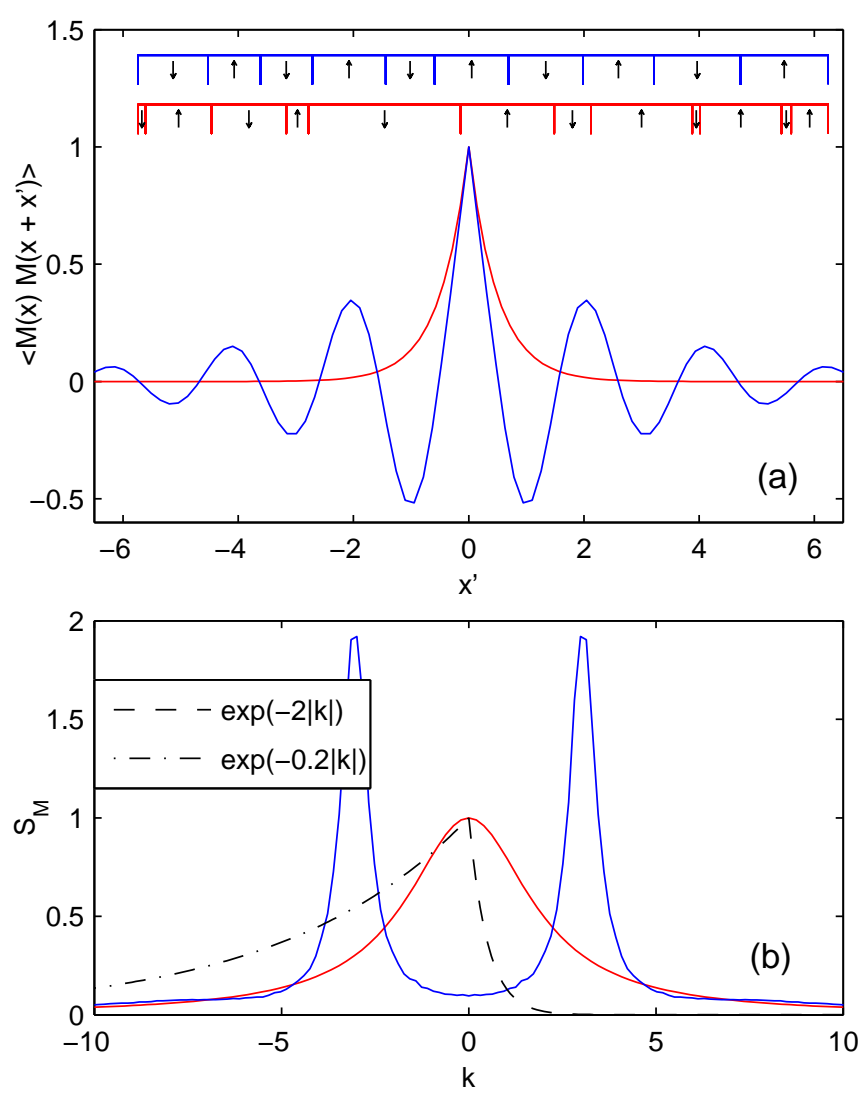

FIG. 1: (Color online) Illustration of different onedimensional domain structures for $|M|=1$. (a) Correlator of $M$, and small sample of the corresponding real space domain structure (inset). (b) Corresponding spectral functions. The domain width in the red (lower) structure is exponentially distributed with mean 1 , which gives $\left\langle M(x) M\left(x+x^{\prime}\right)\right\rangle=e^{-2\left|x^{\prime}\right|}$ and $S_{M}(k)=1 /\left(1+k^{2} / 4\right)$. The domain width in the blue (upper) structure has a Gaussian distribution with mean 1 and standard deviation 0.3, leading to an oscillatory correlator and peaks in the spectral function at $k= \pm \pi$. The curves for this case were obtained numerically from an ensemble of $10^{5}$ domains. The dashed lines in (b) represent the propagation factors $e^{-|k| z}$ for heights $z=2$ and $z=0.2$. For large $z$, only $S_{M}(k \approx 0)$ is relevant.

$S_{M}(0)$ from the observed field variation. Note that if the variation of the domain size is sufficiently large for $\left\langle\mathbf{M}(\mathbf{r}) \mathbf{M}\left(\mathbf{r}+\mathbf{r}^{\prime}\right)\right\rangle$ to be essentially non-negative, as for the exponential width distribution in Fig. 1 $S_{M, \alpha \alpha}(0)=$ $\int d^{3} \mathbf{r}^{\prime}\left\langle M_{\alpha}(\mathbf{r}) M_{\alpha}\left(\mathbf{r}+\mathbf{r}^{\prime}\right)\right\rangle$ can be interpreted as the product of a correlation volume $\int d^{3} \mathbf{r}^{\prime}\left\langle M_{\alpha}(\mathbf{r}) M_{\alpha}(\mathbf{r}+\right.$ $\left.\left.\mathbf{r}^{\prime}\right)\right\rangle /\left\langle M_{\alpha}^{2}\right\rangle$ and the mean square magnetization $\left\langle M_{\alpha}^{2}\right\rangle$.

\section{APPLICATION TO $\mathrm{Sr}_{2} \mathrm{RuO}_{4}$}

Based on various evidence, it is believed that $\mathrm{Sr}_{2} \mathrm{RuO}_{4}$ is a spin triplet superconductor with a $p$-wave order parameter of the same symmetry class as $k_{x} \pm i k_{y}, \underline{5,23}$
Convincing evidence that the order parameter is indeed time-reversal symmetry breaking (TRSB) has recently been obtained by Sagnac-interferometry experiments: 24 Such a TRSB order parameter is expected to cause chiral currents at sample edges, domain walls or impurities $\underline{6.7}$ The direct observation of such effects in $\mathrm{Sr}_{2} \mathrm{RuO}_{4}$ is an ongoing effort. So far, the most direct indication of spontaneous fields is given by $\mu \mathrm{SR}$ data, reporting "a broad distribution of fields arising from a dilute distribution of sources" $\stackrel{25}{\underline{n}}$ Phase sensitive tunneling measurements support the notion of small chiral domains ${ }^{26}$ Scanning Hall probe and scanning SQUID microscopy experiments $\frac{15,16,27}{16}$ on the other hand did not detect any sign of a spontaneous magnetization associated with superconductivity.

The magnetic scans of the $a b$-face in Ref. 15 showed neither localized features nor a random field variation that could be attributed to TRSB. Even holes that were drilled using a focused ion beam (FIB) failed to show a magnetic signature. Lacking suitable theoretical models, a quantitative analysis of those null results was inconclusive. In this section, I will use the results derived above to compute the expected field from domains and defects. This allows to set certain limits on the internal magnetization strength that would be consistent with the data.

A complete description of chiral domains requires a self consistent computation of the order parameter and magnetic fields. This has been carried out using microscopic theory $\underline{\underline{6}}^{\underline{\underline{a}}}$ and a GL approach ${ }^{\underline{7}}$ without considering the effect of a surface. Such detailed calculations generally require a numerical solution. Taking the presence of a surface into account leads to a further complication. Thus, they are rather cumbersome for the purpose of data analysis and planning experiments.

For a domain wall along the $x=0$ plane in an infinite sample, the results of those computations generally show a current along the $y$-direction which decays over about one in-plane coherence length $\xi_{a b}$ in the $x$-direction and changes sign. The counterflowing current decays on the scale of $\lambda$ such that the magnetic field far inside each domain vanishes. Indeed, one can obtain good fits of the form

$$
B_{0, z}(x)=\frac{B_{0}}{1-\tilde{\xi}^{2} / \tilde{\lambda}^{2}} \operatorname{sgn}(x)\left(e^{-|x| / \tilde{\lambda}}-e^{-|x| / \tilde{\xi}}\right)
$$

to the numerical results for the magnetic field of Ref. 6 , with $\tilde{\lambda}=2.2 \xi_{a b}, \tilde{\xi}=1.5 \xi_{a b}$, and $B_{0}=87 \mathrm{G}$. I used $\xi_{a b}=$ $66 \mathrm{~nm}$ and $\lambda=150 \mathrm{~nm}^{5}$ to compute the thermodynamic critical field entering the prefactor of the result of Ref. 6. It is easy to show that this expression is a solution to the London equations for $\lambda=\tilde{\lambda}$ in the presence of a chiral current density $j_{y}(x)=-\left(c B_{0} / 4 \pi \tilde{\xi}\right) e^{-|x| / \tilde{\xi}}$. This current can be identified with an internal magnetization $M_{z}=\left(B_{0} / 4 \pi\right) \operatorname{sgn}(x)\left(1-e^{-|x| / \tilde{\xi}}\right)$ in the $z$ direction due to the orbital magnetic moments of the Cooper pairs. The value of $\tilde{\lambda}$ is in good agreement with the value of $\kappa \equiv \lambda / \xi_{a b}=2.5$ assumed in Ref. 6. This suggests that the London approach captures screening effects quite ac- 
curately, and therefore should give a good approximation for the field above a surface. Of course, this will neglect some features in the full GL solutions. For example, the latter show a slight depression of the superfluid density near the domain wall, which is neglected here by assuming a constant $\lambda$. Similar modifications of both the superfluid density and the chiral current density at the surface should be small due to the short $c$-axis coherence length $\xi_{c}=0.05 \xi_{a b}$. The anisotropy is of no consequence because all currents flow along the $a b$-plane so that only $\lambda_{a b}$ matters.

Since the far field of the domain wall only depends on the difference of the asymptotic values of $M$ away from the domain wall, and experimentally $\xi_{a b}=66 \mathrm{~nm}<\lambda=$ $150 \mathrm{~nm} \ll z \approx 1 \mu \mathrm{m}$, it is appropriate to use the large$z$ results for a sharp discontinuity in $M$ to analyze the results of Ref. 15. If accurate results at a $z \lesssim 3 \lambda$ are required, Eq. (4) together with the above approximation for $B_{0, z}(x)$ should be used. Fig. 2 shows the field profiles for those two methods and the exact result for a sharp domain wall at different heights.

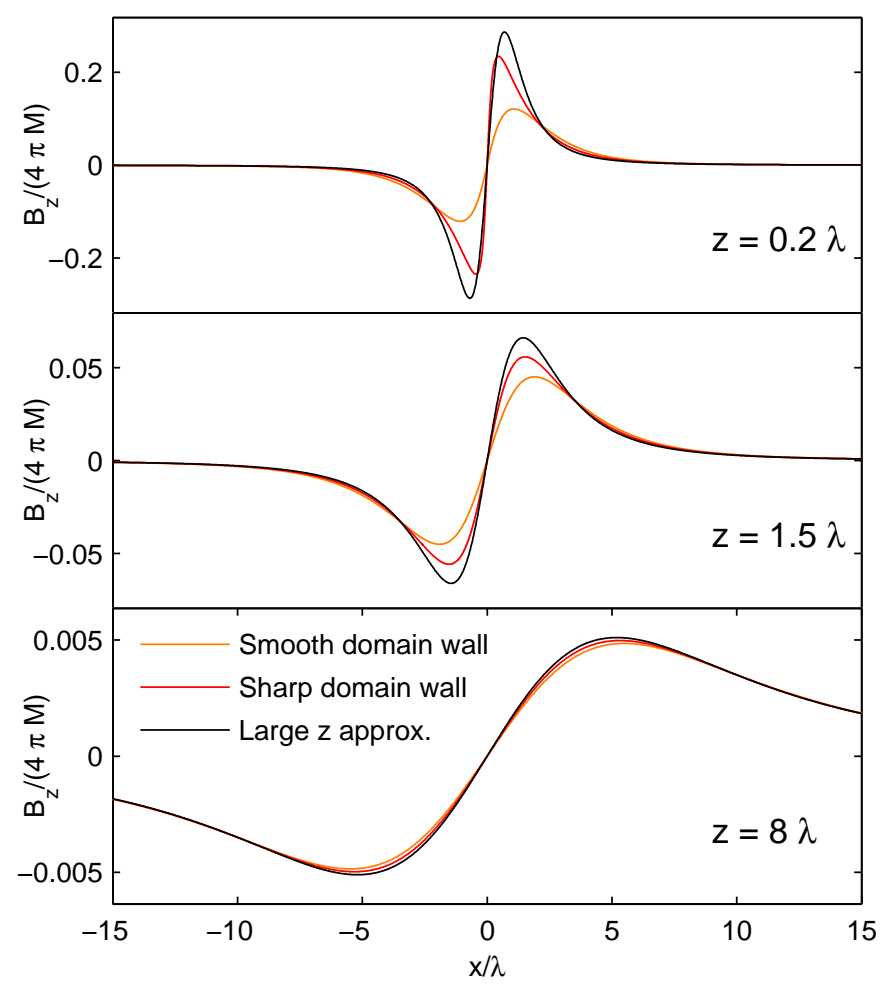

FIG. 2: (Color online) Field profile at different heights $z$ above a domain wall were $M_{z}$ changes sign. The curves labeled "smooth" and "sharp domain wall" were obtained from the full solution with $M_{z}(x)=M \operatorname{sgn}(x)\left(1-e^{-|x| /(0.7 \lambda)}\right)$ and $M_{z}(x)=M \operatorname{sgn}(x)$ respectively. The latter solution and the corresponding large $z$ approximation as shown are given by table I case (2). The value of the domain boundary width was chosen according to the fit to the numerical results for $\mathrm{Sr}_{2} \mathrm{RuO}_{4}$ from Ref. 6. $z=8 \lambda$ corresponds to the Hall probe scans from Ref. 15.
In the experiments, the rms noise levels were $35 \mathrm{mG}$ and $0.45 \mathrm{mG}$ at imaging heights $z=1.2 \mu \mathrm{m}$ and 2 $\mu \mathrm{m}$ in the Hall probe and SQUID scans, respectively. Correction factors due to oversampling and averaging over the size of the SQUID pickup loop are of order unity. An isolated domain wall would result in a field of $5 \cdot 10^{-3} 4 \pi M=0.4 \mathrm{G}$ and $2 \cdot 10^{-3} 4 \pi M=0.17 \mathrm{G}$ respectively at the above imaging heights and $B_{0}=87 \mathrm{G}$, and should be clearly visible in the data. Thus, there was either no such domain wall in the scanned area, or its magnetization was $4 \pi M<7 \mathrm{G}$ and $4 \pi M<0.2 \mathrm{G}$ respectively, so that it was hidden by sensor noise.

The exact calculation of the signature of a hole is more difficult because the translational invariance of the boundary conditions is broken. However, if the diameter of the hole or defect, which I assume to extend along the $z$-direction normal to the surface, is much smaller than $\lambda$, the absence of superfluid in it can be neglected and the dipole calculation should be a good approximation. For a hole or defect with a volume of $\xi_{a b}^{3}$, the maximum field according to case (3) in table पat $z=1.2 \mu \mathrm{m}$ and $2 \mu \mathrm{m}$ is $1.2 \cdot 10^{-5} 4 \pi M=1.1 \mathrm{mG}$ and $2 \cdot 10^{-6} 4 \pi M=0.17 \mathrm{mG}$, respectively. This signal would be nearly undetectable at the experimental noise level of Ref. 15. Furthermore, the extent of the defect along the $z$-direction could be as small as $\xi_{c}=0.05 \xi_{a b}$, and the order parameter is not necessarily suppressed entirely. For a columnar defect on the other hand, one factor of $\xi_{a b}$ has to be replaced by $\lambda$, decreasing the limit on $M$ only by about a factor three. Since the FIB drilled holes in the experiment were significantly larger (about $1 \mu \mathrm{m}$ ), they have both a larger moment and less effective Meissner screening. This leads to a stronger signal whose calculation goes beyond the scope of this paper.

One can also estimate the signal expected from a random configuration of small domains. The smallest conceivable domain volume is on the order of $\xi_{a b}^{2} \xi_{c}$. Assuming that the domain size fluctuates enough to use this as correlation volume, Eq. (15) implies that the rms signal could be as small as $0.7 \mathrm{mG}$ and $0.16 \mathrm{mG}$, again less than the experimental noise. A domain size distribution that does not satisfy the assumptions leading to Eq. (15) may result in even smaller signals. Thus, the possibility of very small domains cannot be ruled out.

In all the above cases, the smaller imaging height of the Hall probe does not compensate for its large noise compared to the SQUID. Assuming the predicted magnitude of the chiral currents $\underline{\underline{6}}$ is correct, the calculations show that any domain wall should have been detected by the measurements. Small defects on the other hand might easily have been hidden in the noise. It is also possible that a random signal from domains would have been too small to observe, especially if the domains are short in the $c$-direction or very homogeneous in size while not too large. However, it appears that one should not take the notion of a magnetization due to $p$-wave pairing too literally. It was shown 28 that the chiral currents can in general not be written as the curl of a global magneti- 
zation, and that the effective value of $\mathbf{M}$ depends on the type of domain wall.

Note that a similar analysis of the magnetic scanning data of Refs. 15, 16, also making use of the relations derived in Sec. III has been carried out in parallel with the present work in Ref. 16 .

\section{CONCLUSION}

I have presented a model for a superconductor with an intrinsic magnetization by combining the macroscopic magnetostatic Maxwell equation with the London relation and obtained the field geometry at a planar superconductor-vacuum interface for a given spatial variation of the magnetization. Solutions for a range of specific magnetic domain boundary configurations give simple expressions in the limit of a large height above the sample. If the height above the surface at which the magnetic field can be measured exceeds the characteristic length scale of variations in the magnetization, a spectral analysis can be used to relate the spectral densities of the two at resolvable wave vectors. If a specific model for the structure of the magnetization is at hand, a direct comparison with the measured field is possible. Otherwise, some simplifying assumptions give a simple estimate relating the spectral density of $\mathbf{M}$ at the superconductor vacuum interface to the observable spatial rms-variation of the magnetic field. As an example for an application, I have applied my calculations to recent experimental results on $\mathrm{Sr}_{2} \mathrm{RuO}_{4}{ }^{15}$, concluding that large chiral domains would have been visible in those experiments, but small domains and defects may have escaped detection.

\section{Acknowledgments}

I would like to thank Kam Moler, Ophir Auslaender and John Kirtley for giving feedback on the manuscript. This work has been supported by the Department of Energy under contract DE-AC02-76SF00515.
* Electronic address: hendrikb@stanford.edu

1 J. W. Lynn, D. E. Moncton, W. Thomlinson, G. Shirane, and R. N. Shelton, Solid State Commun. 26, 493 (1978).

2 M. Ishikawa and O. Fischer, Solid State Commun. 23, 37 (1977).

3 W. A. Fertig, D. C. Johnston, L. E. DeLong, R. W. McCallum, M. B. Maple, and B. T. Matthias, Phys. Rev. Lett. 38, 987 (1977).

4 Y. Maeno, H. Hashimoto, K. Yoshida, S. Nishizaki, T. Fujita, J. G. Bednorz, and F. Lichtenberg, Nature (London) 372, 532 (1994).

5 A. P. Mackenzie and Y. Maeno, Rev. Mod. Phys. 75, 657 (2003), and references therein.

6 M. Matsumoto and M. Sigrist, J. Phys. Soc. J. 68, 994 (1999), see also erratum in Vol. 68, p. 3120.

7 M. Sigrist, T. M. Rice, and K. Ueda, Phys. Rev. Lett. 63, 1727 (1989)

8 G. Baskaran, Phys. Rev. Lett. 91, 097003 (2003).

9 A. M. Chang, H. D. Hallen, L. Harriott, H. F. Hess, H. L. Kao, J. Kwo, R. E. Miller, R. Wolfe, J. Vanderziel, and T. Y. Chang, Applied Physics Letters 61, 1974 (1992).

10 J. R. Kirtley and J. P. Wikswo, Annual Review of Materials Science 29, 117 (1999).

11 D. Rugar, H. J. Mamin, P. Guethner, S. E. Lambert, J. E. Stern, I. McFadyen, and T. Yogi, J. Appl. Phys. 68, 1169 (1990).

12 A. Moser, H. J. Hug, I. Parashikov, B. Stiefel, O. Fritz, H. Thomas, A. Baratoff, H. J. Guntherodt, and P. Chaudhari, Phys. Rev. Lett. 74, 1847 (1995).

13 M. R. Koblischka and R. J. Wijngaarden, Superconductor Science \& Technology 8, 199 (1995).

14 H. Bluhm, S. E. Sebastian, J. W. Guikema, I. R. Fisher, and K. A. Moler, Phys. Rev. B 73, 14514 (2006).

15 P. G. Bjornsson, Y. Maeno, M. E. Huber, and K. A. Moler, Phys. Rev. B 72, 012504 (2005).

16 J. Kirtley, C. Kallin, C. Hicks, E.-A. Kim, Y. Liu, K. Moler, Y. Maeno, and K. Nelson, arXiv:0704.3364v1 [cond-mat.supr-con] (2007), (unpublished).

17 H. S. Greenside, E. I. Blount, and C. M. Varma, Phys. Rev. Lett. 46, 49 (1981).

18 E. I. Blount and C. M. Varma, Phys. Rev. Lett. 42, 1079 (1979).

19 T. K. Ng and C. M. Varma, Phys. Rev. Lett. 78, 330 (1997)

20 C. G. Kuper, M. Revzen, and A. Ron, Phys. Rev. Lett. 44, 1545 (1980).

21 E. Morenzoni, T. Prokscha, A. Suter, H. Luetkens, and R. Khasanov, J. Phys. Cond. Mat. 16, S4583 (2004).

${ }^{22}$ V. G. Kogan, A. Y. Simonov, and M. Ledvij, Phys. Rev. B 48, 392 (1993).

23 K. D. Nelson, Z. Q. Mao, Y. Maeno, and Y. Liu, Science 306, 1151 (2004).

24 J. Xia, Y. Maeno, P. T. Beyersdorf, M. M. Fejer, and A. Kapitulnik, Phys. Rev. Lett. 97, 167002 (2006).

${ }^{25}$ G. M. Luke, Y. Fudamoto, K. M. Kojima, M. I. Larkin, J. Merrin, B. Nachumi, Y. J. Uemura, Y. Maeno, Z. Q. Mao, Y. Mori, et al., Nature 394, 558 (1998).

${ }^{26}$ F. Kidwingira, J. D. Strand, D. J. V. Harlingen, and Y. Maeno, Science 314, 1267 (2006).

27 V. O. Dolocan, C. Veauvy, F. Servant, P. Lejay, K. Hasselbach, Y. Liu, and D. Mailly, Phys. Rev. Lett. 95, 097004 (2005).

28 V. Braude and E. B. Sonin, Phys. Rev. B 74, 64501 (2006). 